\title{
Factor Analysis of Low-Frequency Repetitive Transcranial Magnetic Stimulation to the Temporoparietal Junction for Tinnitus
}

\author{
Hui Wang, ${ }^{1}$ Bei Li, ${ }^{1}$ Meiye Wang, ${ }^{1}$ Ming Li, ${ }^{2}$ Dongzhen Yu, ${ }^{1}$ Haibo Shi, ${ }^{1}$ and Shankai Yin ${ }^{1}$ \\ ${ }^{1}$ Department of Otolaryngology Head and Neck Surgery, Shanghai Jiao Tong University Affiliated Sixth People's Hospital, \\ Shanghai 200233, China \\ ${ }^{2}$ Department of Otolaryngology, Tinnitus and Hyperacusis Center Yueyang Hospital of Integrated Traditional Chinese and \\ Western Medicine Affiliated to Shanghai University of Traditional Chinese Medicine, Shanghai 200437, China
}

Correspondence should be addressed to Dongzhen Yu; doctor.yu@126.com and Haibo Shi; haibo99@hotmail.com

Received 5 July 2016; Revised 16 September 2016; Accepted 3 October 2016

Academic Editor: Genglin Li

Copyright (C) 2016 Hui Wang et al. This is an open access article distributed under the Creative Commons Attribution License, which permits unrestricted use, distribution, and reproduction in any medium, provided the original work is properly cited.

Objectives. We investigated factors that contribute to suppression of tinnitus after repetitive transcranial magnetic stimulation (rTMS). Methods. A total of 289 patients with tinnitus underwent active $1 \mathrm{~Hz}$ rTMS in the left temporoparietal region. A visual analog scale (VAS) was used to assess tinnitus loudness. All participants were interviewed regarding age, gender, tinnitus duration, laterality and pitch, audiometric parameters, sleep, and so forth. The resting motor thresholds (RMTs) were measured in all patients and 30 age- and gender-matched volunteers. Results. With respect to different factors that contribute to tinnitus suppression, we found improvement in the following domains: shorter duration, normal hearing (OR: 3.25, 95\%CI: 2.01-5.27, $p=0.001$ ), and without sleep disturbance (OR: 2.51,95\%CI: 1.56-4.1, $p=0.005$ ) adjusted for age and gender. The patients with tinnitus lasting less than 1 year were more likely to show suppression of tinnitus (OR: 2.77, 95\%CI: 1.48-5.19, $p=0.002$ ) compared to those with tinnitus lasting more than 5 years. Tinnitus patients had significantly lower RMTs compared with healthy volunteers. Conclusion. Active low-frequency rTMS results in a significant reduction in the loudness of tinnitus. Significant tinnitus suppression was shown in subjects with shorter tinnitus duration, with normal hearing, and without sleep disturbance.

\section{Introduction}

Tinnitus is a subjective, mostly transitory, phantom auditory perception of sound that affects millions of people at some point in their lives [1]. It is estimated that tinnitus will become chronic and severely affect the quality of life in $1 \%-3 \%$ of the general population [2]. About $20 \%$ of adults that experience tinnitus will require clinical intervention [3]. Tinnitus can occur constantly or intermittently in one or both ears or centrally in the head and can be perceived as coming from within the head. There is convincing evidence from functional imaging and neurophysiological studies that central mechanisms are responsible for most cases of tinnitus [46], which may be caused by (1) changes in the firing pattern of neurons in the central auditory system, (2) changes in burst firing and neural synchrony, and (3) cortical tonotopic map reorganization [7]. All of the above may occur due to alterations in neuronal activity in the brain cortex, which suggests promising treatment strategies for tinnitus.

Based on the above findings, repetitive transcranial magnetic stimulation (rTMS) of the temporal and temporoparietal cortex has been proposed as a promising treatment for chronic tinnitus. Its mechanism of action involves targeting the hyperactivity/abnormal synchronization within the auditory cortex to suppress tinnitus [8-10]. rTMS temporarily disrupts a circumspect area of the cortex that interrupts normal functioning and has acute and chronic effects in tinnitus patients. Several clinical studies consistently showed a reduction of tinnitus severity after application of rTMS to the left temporoparietal region of the cortex [11-13]. However, the treatment results showed high interindividual variability $[14,15]$, indicating the need for optimization of the 
indications. This variability also indicates that a large sample study is needed.

The present study was using active rTMS performed to investigate optimization of the indications by evaluating the different factors that contribute to tinnitus suppression after low-frequency rTMS for the treatment of chronic tinnitus.

\section{Methods}

2.1. Subjects. Patients suffering from nonpulsatile and constant tinnitus examined and treated at the Affiliated Sixth People's Hospital Otolaryngology Department, Shanghai Jiao Tong University, between December 2014 and December 2015, were included in this study. With local ethics committee approval, written informed consent was obtained from all subjects prior to enrollment, and the possible consequences of the study were explained. Our registration number is ChiCTR-INR-16008092. Upon recruitment, the subjective tinnitus loudness perception in patients was determined using a visual analog scale (VAS) ranging from 0 to 10 , where 0 indicates no tinnitus and 10 indicates the worst possible tinnitus-related discomfort. VAS improvement in responders decreased to $20 \%$ of the basal score indicating no reduction, $40 \%, 60 \%$, and $80 \%$ of the basal score indicating slight, marked, and strong reduction, and $100 \%$ reduction indicating complete suppression of tinnitus immediately after stimulation. The reduction range from $40 \%$ to $100 \%$ is considered as "good effect." Measurements were made before, immediately, and 2 weeks after the last intervention session.

Detailed histories were obtained from all patients, including a clinical examination and audiogram. Hearing level was assessed by using an audiometer in a soundproof room, and the loudness and pitch of tinnitus were evaluated with a TinniTest audiometer. Normal hearing was defined as normal audiogram (threshold $<25 \mathrm{~dB} \mathrm{HL}$ at all frequencies from 0.25 to $8 \mathrm{kHz}$ ) as well as a normal tympanometric curve of type A. The ipsilateral and contralateral stapedial reflexes were also included. All participants were comprehensively interviewed for information regarding their age, gender, tinnitus duration, tinnitus laterality, pitch of tinnitus, sleep quality, accompanying symptoms, and so forth.

2.2. rTMS Procedure. All of the patients underwent rTMS over the left temporoparietal cortex region. As described in our previous study [6], rTMS consisted of 1000 stimuli at $1 \mathrm{~Hz}$ daily and $110 \%$ of the motor cortex threshold for 5 consecutive days per week (Monday to Friday) for 2 weeks. For repetitive pulses, TMS was delivered through a focal figure-eight magnetic coil connected to a magnetic stimulator (MagPro R30; MagVenture, Farum, Denmark). The resting motor threshold (RMT) was defined as the lowest stimulator output intensity capable of inducing motor evoked potentials (MEPs) of at least $50 \mu \mathrm{V}$ peak-to-peak amplitude in the relaxed state in at least 5 of 10 consecutive trials. Thirty volunteers with normal hearing served as controls, whose RMTs were obtained. The tinnitus subjects and controls were age- and gender-matched.

2.3. Statistical Analysis. All statistical analyses were performed using SPSS v 16.0 (SPSS, Chicago, IL, USA). Prior to analysis, all variables were examined for their normality. All categorical variables were analyzed by the Chi-square test. We calculated the odds ratio (OR) and associated 95\% confidence interval (CI) for demographic factors using the Mantel-Haenszel method. Logistic regression was performed to identify the factors contributing to tinnitus suppression after the intervention. In all analyses, $p<0.05$ was taken to indicate statistical significance.

\section{Results}

The stimulation protocols were well tolerated, and all the patients completed the treatment except three patients who reported transient mild to moderate headache, two patients who reported transient worsening of their tinnitus, and two patients that complained of vertigo. None of the patients developed seizures or other serious side effects or adverse effects. A total of 289 patients with chronic unilateral or bilateral tinnitus (137 male, 182 female, age range: 19-87 years, mean $57 \pm 14.7$ years) were included in the study. In the active stimulation group, $76(26.3 \%)$ patients reported purely left-sided tinnitus, 50 (17.3\%) patients reported purely rightsided tinnitus, 129 (44.6\%) patients described their tinnitus as bilateral, and $34(11.8 \%)$ patients described their tinnitus as originating within the head. The duration of tinnitus ranged from 7 months to 40 years, with a median of 5.8 years, and approximately $15.2 \%$ of the subjects had experienced tinnitus for more than 5 years. The patients rated their tinnitus loudness on the VAS, and the median score was 6.5 (range, $4-10)$. A total of $169(58.5 \%)$ of the 289 patients reported tonal tinnitus and 108 (37.4\%) patients described noisiform tinnitus. Twelve (4.2\%) patients could not determine the pitch of tinnitus or their pitch of tinnitus was unmatchable. Audiometric assessment showed normal hearing in 143 of 289 subjects (49.5\%) and hearing loss in 146 (50.5\%). Tinnitus interfered with sleep in 129 of the 289 patients.

The clinical characteristics of the tinnitus patients are shown in Table 1. Participants completed a questionnaire assessment of medical history before and immediately after the last intervention. Information on age, gender, tinnitus laterality, duration of tinnitus, hearing level, pitch of tinnitus, accompanying symptoms, underlying diseases, and sleep quality was included in the analyses.

rTMS showed a good effect in 138 of the patients included in the study (47.8\%) and no effect in 151 patients (52.2\%) in the active group. With respect to different factors contributing to tinnitus suppression, we observed improvement after active rTMS in the following domains: age, gender, duration of tinnitus, tinnitus laterality, audiometric parameters, and sleep. Significant tinnitus suppression was associated with younger age, male gender, shorter duration of tinnitus, tinnitus located centrally in the head, normal hearing, and no sleep disturbance at night $(p<0.05)$. These patients showed no significant differences in pitch of tinnitus, accompanying symptoms, such as headache and vertigo, and underlying diseases, such as hypertension, diabetes, and heart disease $(p>0.05)$ (Table 1).

Before the intervention, the average baseline VAS score in patients with active stimulation was 5.5. The score was 
TABLE 1: Clinical characteristics of the patients suffering from tinnitus.

\begin{tabular}{|c|c|c|c|c|}
\hline \multirow{2}{*}{ Independent variables } & \multicolumn{2}{|c|}{ Active group } & \multirow{2}{*}{$N$} & \multirow{2}{*}{$p$ value } \\
\hline & No effect $\%$ & Improved $\%$ & & \\
\hline \multicolumn{5}{|l|}{ Age (yrs) } \\
\hline$<30$ & $5(29.41)$ & $12(70.59)$ & 17 & \multirow{4}{*}{0.012} \\
\hline $31-50$ & $30(48.39)$ & $32(51.61)$ & 62 & \\
\hline $51-70$ & $88(51.16)$ & $84(48.84)$ & 172 & \\
\hline$>70$ & $28(73.68)$ & $10(26.32)$ & 38 & \\
\hline \multicolumn{5}{|l|}{ Gender } \\
\hline Male & $56(45.16)$ & $68(54.84)$ & 124 & \multirow{2}{*}{0.043} \\
\hline Female & $95(57.58)$ & $70(42.42)$ & 165 & \\
\hline \multicolumn{5}{|l|}{ Underlying diseases } \\
\hline Good condition & $121(50.21)$ & $120(49.79)$ & 241 & \multirow{2}{*}{0.154} \\
\hline Underlying diseases & $30(62.50)$ & $18(37.50)$ & 48 & \\
\hline \multicolumn{5}{|l|}{ Accompanied symptoms } \\
\hline No accompanied symptoms & s $95(47.74)$ & $104(52.26)$ & 199 & \multirow{3}{*}{0.052} \\
\hline Headache & $18(69.23)$ & $8(30.77)$ & 26 & \\
\hline Dizziness & $38(59.38)$ & $26(40.62)$ & 64 & \\
\hline \multicolumn{5}{|l|}{ Pitch of tinnitus } \\
\hline Tone & $79(46.75)$ & $90(53.25)$ & 169 & \multirow{3}{*}{0.06} \\
\hline Noise & $66(61.11)$ & $42(38.89)$ & 108 & \\
\hline Uncertainty & $6(50.00)$ & $6(50.00)$ & 12 & \\
\hline \multicolumn{5}{|l|}{ Tinnitus laterality } \\
\hline Left ear & $38(50.00)$ & $38(50.00)$ & 76 & \multirow{4}{*}{0.025} \\
\hline Right ear & $28(56.00)$ & $22(44.00)$ & 50 & \\
\hline Bilateral & $75(58.14)$ & $54(41.86)$ & 129 & \\
\hline Ringing in the head & $10(29.41)$ & $24(70.59)$ & 34 & \\
\hline \multicolumn{5}{|l|}{ Audiometric parameters } \\
\hline Normal hearing & $53(37.59)$ & $88(62.41)$ & 141 & \multirow{2}{*}{0.001} \\
\hline Sensorineural hearing loss & $98(66.22)$ & $50(33.78)$ & 148 & \\
\hline \multicolumn{5}{|l|}{ Tinnitus duration } \\
\hline$\leq 1 \mathrm{yr}$ & $29(39.73)$ & $44(60.27)$ & 73 & \multirow{4}{*}{0.013} \\
\hline $1 \mathrm{yr}<x \leq 2 \mathrm{yrs}$ & $26(48.15)$ & $28(51.85)$ & 54 & \\
\hline $2 \mathrm{yrs}<x \leq 5 \mathrm{yrs}$ & $62(64.58)$ & $34(35.42)$ & 96 & \\
\hline$>5 \mathrm{yrs}$ & $34(51.51)$ & $32(48.49)$ & 66 & \\
\hline \multicolumn{5}{|l|}{ Sleep } \\
\hline Sleep disturbance & $84(64.62)$ & $46(35.38)$ & 130 & \multirow{2}{*}{0.005} \\
\hline Sleep well & $67(42.14)$ & $92(57.86)$ & 159 & \\
\hline
\end{tabular}

decreased to 2.7 immediately after the last active intervention. After active rTMS, 51.3\% (SD = 20.6\%) patients experienced a significant reduction in tinnitus loudness, as evidenced by VAS scale.

In the present study, the duration of tinnitus was correlated with tinnitus suppression by rTMS. The intervention was more effective in suppressing tinnitus in patients that had suffered from tinnitus for only a short time. Multinomial logistic regression analysis was performed with tinnitus duration as a dependent variable and the change in VAS score before and after stimulation as a predictor variable. There
TABLE 2: Multivariate stepwise logistic regression analyses models for different factors that contribute to tinnitus suppression.

\begin{tabular}{|c|c|c|c|}
\hline $\begin{array}{l}\text { Independent } \\
\text { variables }\end{array}$ & Odds ratio & $\begin{array}{c}95 \% \\
\text { Confidence interval }\end{array}$ & $p$ value \\
\hline \multicolumn{4}{|l|}{ Age (yrs) } \\
\hline$<30$ & 0.24 & $0.06-1.07$ & $p=0.061$ \\
\hline $31-50$ & 0.48 & $0.14-1.69$ & $p=0.255$ \\
\hline $51-70$ & 0.29 & $0.08-1.12$ & $p=0.072$ \\
\hline$>70$ & 1 & & \\
\hline \multicolumn{4}{|l|}{ Gender } \\
\hline Female & 1 & & \\
\hline Male & 1.57 & $0.88-2.76$ & $p=0.122$ \\
\hline \multicolumn{4}{|l|}{ Tinnitus laterality } \\
\hline Left ear & 1 & & \\
\hline Right ear & 0.81 & $0.36-1.81$ & $p=0.602$ \\
\hline Bilateral & 0.85 & $0.45-1.60$ & $p=0.613$ \\
\hline $\begin{array}{l}\text { Ringing in the } \\
\text { head }\end{array}$ & 1.63 & $0.62-4.31$ & $p=0.324$ \\
\hline \multicolumn{4}{|l|}{ Hearing level } \\
\hline Normal hearing & 3.25 & $2.01-5.27$ & $p=0.001$ \\
\hline $\begin{array}{l}\text { Sensorineural } \\
\text { hearing loss }\end{array}$ & 1 & & \\
\hline \multicolumn{4}{|l|}{ Duration of tinnitus } \\
\hline$>5 \mathrm{yrs}$ & 1 & & \\
\hline $2 \mathrm{yrs}<x \leq 5 \mathrm{yrs}$ & 1.52 & $0.70-3.10$ & $p=0.441$ \\
\hline $1 \mathrm{yr}<x \leq 2 \mathrm{yrs}$ & 1.61 & $0.82-3.20$ & $p=0.731$ \\
\hline$\leq 1 \mathrm{yrs}$ & 2.77 & $1.48-5.19$ & $p=0.002$ \\
\hline \multicolumn{4}{|l|}{ Sleep } \\
\hline Sleep disturbance & 1 & & \\
\hline $\begin{array}{l}\text { Without sleep } \\
\text { disturbance }\end{array}$ & 2.51 & $1.56-4.10$ & $p=0.005$ \\
\hline
\end{tabular}

was a linear (negative) correlation between the duration of tinnitus and the degree of tinnitus suppression by rTMS ( $p=$ 0.013 ). Patients with tinnitus lasting less than 1 year were more likely to show suppression of tinnitus (OR: $2.77,95 \%$ CI: $1.48-5.19, p=0.002$ ) immediately after the last intervention compared to those with tinnitus lasting more than 5 years (Table 2). Tinnitus could be suppressed, on average, in $60.2 \%$ of patients with tinnitus lasting less than 1 year but only in $51.8 \%$ of those with symptom duration of less than 2 years and only in $40.7 \%$ of those with symptoms lasting for more than 2 years.

When compared to patients with sensorineural hearing loss, those with normal hearing were more likely to show suppression of tinnitus (OR: 3.25, 95\% CI: 2.01-5.27, $p=$ 0.001 ) immediately after the last intervention. In addition, the rTMS treatment showed a significant effect in patients that reported no sleep disturbance (OR: 2.51, 95\% CI: 1.56-4.10, $p=0.005)$. Patients with tinnitus located centrally in the head often reported a reduction in tinnitus after the rTMS procedure in contrast to those with left or right tinnitus (OR: 1.63, 95\% CI: $0.62-4.31 p=0.324)$. Although rTMS was 

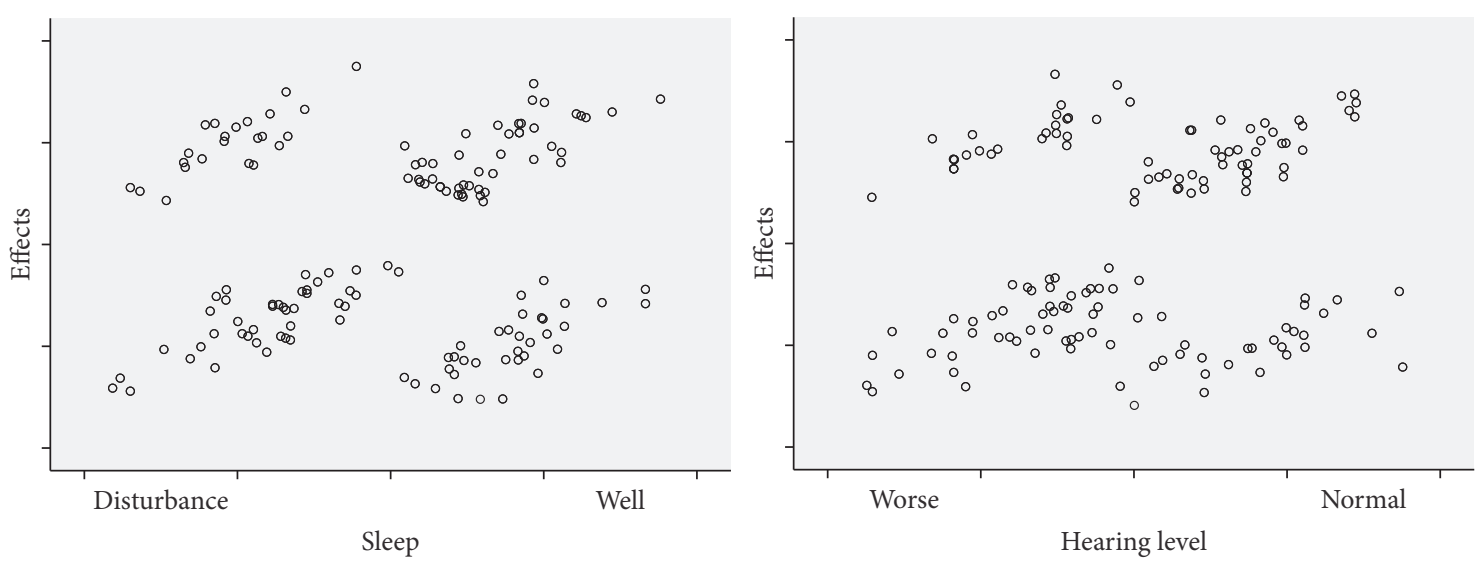

FIGURE 1: Correlations were observed between sleep and hearing level and efficacy of rTMS. The intervention was more likely to suppress tinnitus in subjects with normal hearing and those without sleep disturbance.

only applied over the left temporoparietal cortex, tinnitus was decreased equally well regardless of whether it was predominantly experienced in the left or right ear. There was no significant difference in the outcome between ipsilateral and contralateral rTMS. There was no significant difference in the outcome between bilateral and left-sided stimulation either (Table 2).

To investigate the major determinants of the effects of rTMS on tinnitus, we performed stepwise linear regression analyses between baseline characteristics, such as age, sex, duration of tinnitus, tinnitus laterality, hearing level, sleep quality, and efficacy of treatment. Age, gender, duration of tinnitus, hearing level, and sleep quality showed significant associations with the efficacy of rTMS, while tinnitus laterality showed no considerable effect ( $p=0.233)$. On multiple regression analysis adjusted for age and gender, the patients with shorter tinnitus duration and normal hearing and without sleep disturbance showed maximal induction of tinnitus suppression by rTMS (Figure 1).

Baseline RMT in tinnitus patients was $48.5 \%$, and that in healthy controls was $53.1 \%$, indicating that tinnitus patients had significantly lower RMTs (Figure 2 ) than healthy volunteers $(t=2.926, p=0.004)$.

\section{Discussion}

The present study demonstrated that active low-frequency rTMS results in a significant decrease in the loudness of tinnitus. With respect to different factors that contribute to tinnitus suppression, active rTMS induces maximal tinnitus suppression in subjects with shorter tinnitus duration, normal hearing, and without sleep disturbance. The tinnitus patients have enhanced brain excitability compared with the controls.

The use of rTMS in the treatment of tinnitus stems from the development of models of central generation induced by auditory deafferentation (neural plasticity with hypersynchrony or hyperactivity of cortical and subcortical auditory and nonauditory areas) [16-19]. The results of the present study showed that tinnitus can be transiently suppressed

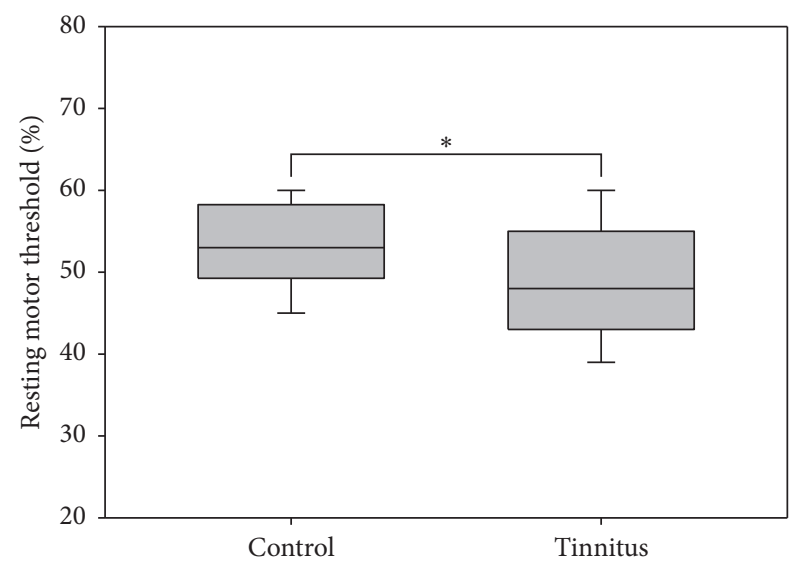

FIGURE 2: The resting motor threshold was significantly lower in tinnitus patients compared with healthy volunteers.

partially or completely by rTMS in approximately $47.8 \%$ of tinnitus patients. Tinnitus duration, hearing level, and sleep quality were identified as positive predictors, in agreement with previous studies [20]. The amount of tinnitus suppression by rTMS was inversely correlated with the duration of symptoms; that is, shorter duration of tinnitus was associated with a greater suppressive effect. Two years seems to be an important turning point for obtaining a beneficial outcome. Tinnitus lasting less than 1 year could be suppressed on average in $60.2 \%$ of responding patients, decreasing to $51.8 \%$ of those with a symptom duration of less than 2 years and $40.7 \%$ after more than 2 years. It is possible that the central network involved in tinnitus becomes less plastic and less responsive to rTMS intervention over time. In addition, the degree of tinnitus suppression achieved through rTMS depended on the hearing of the patients. Hearing impairment was also identified as a negative predictor in our study. When compared to patients with sensorineural hearing loss, those with normal hearing were more likely to show suppression of tinnitus immediately after the last intervention. These observations suggested that hearing loss may represent an ongoing trigger for the generation of tinnitus and both reduce and 
shorten the TMS treatment effects. In addition, the deprivation of auditory input may lead to disinhibition in the central auditory system, which in turn may exacerbate the plastic changes in neural functioning that could underlie tinnitus [21]. In addition, the reduction of inhibition in central auditory structures leads to hyperexcitability of circumscribed regions of the central auditory system, as evidenced by the enhanced brain excitability indicated in the present study. Moreover, rTMS treatment had a significant effect in patients without sleep disturbance. Nonauditory areas, such as the frontoparietal areas and limbic areas, have been suggested to be involved in the pathophysiology of tinnitus, as evidenced by the functional imaging data in our previous study [6]. These interactions between auditory and nonauditory brain regions may explain why tinnitus is perceived as bothersome. These mechanisms can lead to comorbid conditions, such as concentration problems, depression, and sleep disturbances [22]. Accordingly, impaired sleep quality shows an increased prevalence in cases of chronic tinnitus. In addition, patients frequently report that tinnitus prevents them from falling asleep.

Low-frequency rTMS, which is known to suppress cortical excitability, has been used successfully to interfere with neural functioning in the temporoparietal cortical region as the magnetic field passes through the skull and induces a small secondary current in the cortex $[9,23]$. Accordingly, low-frequency rTMS has been used to treat tinnitus [24, 25]. In addition, TMS can also be used as a diagnostic tool for the assessment of motor cortex excitability by quantifying contractions of peripheral muscles induced by stimulation of the corresponding motor cortex representation. In the present study, tinnitus patients were shown to have enhanced brain excitability compared with controls, which was consistent with previous studies suggesting that central mechanisms are responsible for at least some cases of tinnitus [26]. The enhanced brain excitability was accompanied by a decrease in glucose metabolism and inhibitory-acting $\gamma$-aminobutyric acid (GABA) in the stimulated temporal cortex and an increase in cingulate and frontal areas but also in motor cortex, as evidenced by the previous study [27-29]. Many efforts have been made to gain insight into the neurophysiological mechanisms of tinnitus [30]. This knowledge can contribute to investigations into the pathophysiology of tinnitus.

Evidence from previous studies suggested that a functional network of several cortical areas may be responsible for tinnitus, but the precise region affected remains unclear $[31,32]$. Using PET imaging, Plewnia et al. [33] found greater activity in the left auditory cortex of chronic tinnitus patients, regardless of the side of symptoms, or centrally within the head. Although rTMS was only applied over the left temporoparietal cortex in the present study, patients responded equally well regardless of whether their symptoms were predominantly in the left or right ear. The rate of response in tinnitus located centrally in the head was even higher than that for tinnitus located predominantly in the ears. However, on multiple regression analysis adjusted for age and gender, tinnitus laterality had no considerable effect.

In conclusion, active low-frequency rTMS resulted in significant suppression of the loudness of tinnitus, especially in patients with a shorter tinnitus duration and normal hearing and without sleep disturbance. Tinnitus patients showed enhanced brain excitability compared with controls. Future studies may significantly benefit from emerging imaging techniques to identify the mechanisms underlying tinnitus and from stimulation protocols that will together determine the optimal site for targeting rTMS stimulation.

\section{Competing Interests}

The authors declare that there are no competing interests.

\section{Authors' Contributions}

Hui Wang, Bei Li, and Meiye Wang have contributed equally to this work.

\section{Acknowledgments}

This study was supported by grants from Excellent Academic Leader Training Program of Shanghai Municipal Health System (XBR2013085), cooperation projects of new perspectives and advanced technology based on shanghai hospitals (SHDC 12014125), and National Natural Science Foundation of China (81570908/H1304).

\section{References}

[1] C. P. Lanting, E. de Kleine, and P. van Dijk, "Neural activity underlying tinnitus generation: results from PET and fMRI," Hearing Research, vol. 255, no. 1-2, pp. 1-13, 2009.

[2] D. E. Tunkel, C. A. Bauer, G. H. Sun et al., "Clinical practice guideline: tinnitus," Otolaryngology-Head and Neck Surgery, vol. 151, no. 2, supplement, pp. S1-S40, 2014.

[3] C. W. Newman, S. A. Sandridge, and G. P. Jacobson, "Assessing outcomes of tinnitus intervention," Journal of the American Academy of Audiology, vol. 25, no. 1, pp. 76-105, 2014.

[4] A. Crippa, C. P. Lanting, P. V. Dijk, and J. B. Roerdink, "A diffusion tensor imaging study on the auditory system and tinnitus," The Open Neuroimaging Journal, vol. 4, pp. 16-25, 2010.

[5] C. P. Lanting, E. de Kleine, R. N. Eppinga, and P. van Dijk, "Neural correlates of human somatosensory integration in tinnitus," Hearing Research, vol. 267, no. 1-2, pp. 78-88, 2010.

[6] H. Wang, B. Li, Y. Feng et al., "A pilot study of EEG source analysis based repetitive transcranial magnetic stimulation for the treatment of tinnitus," PLoS ONE, vol. 10, no. 10, Article ID e0139622, 2015.

[7] A. J. Norena, "Revisiting the cochlear and central mechanisms of tinnitus and therapeutic approaches," Audiology and Neurotology, vol. 20, supplement 1, pp. 53-59, 2015.

[8] O. M. Meeus, D. De Ridder, and P. H. Van de Heyning, "Transcranial magnetic stimulation (TMS) in tinnitus patients," $B$-ENT, vol. 5, no. 2, pp. 89-100, 2009.

[9] I. Lorenz, N. Müller, W. Schlee, B. Langguth, and N. Weisz, "Short-term effects of single repetitive TMS sessions on auditory evoked activity in patients with chronic tinnitus," Journal of Neurophysiology, vol. 104, no. 3, pp. 1497-1505, 2010. 
[10] J. P. Lefaucheur, N. André-Obadia, A. Antal et al., "Evidencebased guidelines on the therapeutic use of repetitive transcranial magnetic stimulation (rTMS)," Clinical Neurophysiology, vol. 125, no. 11, pp. 2150-2206, 2014.

[11] S. Vanneste and D. De Ridder, "The involvement of the left ventrolateral prefrontal cortex in tinnitus: a TMS study," Experimental Brain Research, vol. 221, no. 3, pp. 345-350, 2012.

[12] S. Rossi, A. De Capua, M. Ulivelli et al., "Effects of repetitive transcranial magnetic stimulation on chronic tinnitus: a randomised, crossover, double blind, placebo controlled study," Journal of Neurology, Neurosurgery and Psychiatry, vol. 78, no. 8, pp. 857-863, 2007.

[13] A. Lehner, M. Schecklmann, T. B. Poeppl et al., "Multisite rTMS for the treatment of chronic tinnitus: stimulation of the cortical tinnitus network-a pilot study," Brain Topography, vol. 26, no. 3, pp. 501-510, 2013.

[14] A. Londero, B. Langguth, D. De Ridder, P. Bonfils, and J.P. Lefaucheur, "Repetitive transcranial magnetic stimulation (rTMS): a new therapeutic approach in subjective tinnitus?" Neurophysiologie Clinique, vol. 36, no. 3, pp. 145-155, 2006.

[15] J. Burger, E. Frank, P. Kreuzer et al., "Transcranial magnetic stimulation for the treatment of tinnitus: 4-year follow-up in treatment responders-a retrospective analysis," Brain Stimulation, vol. 4, no. 4, pp. 222-227, 2011.

[16] B. Langguth, P. Eichhammer, R. Wiegand et al., "Neuronavigated rTMS in a patient with chronic tinnitus. Effects of 4 weeks treatment,” NeuroReport, vol. 14, no. 7, pp. 977-980, 2003.

[17] C. Plewnia, M. Bartels, and C. Gerloff, “Transient suppression of tinnitus by transcranial magnetic stimulation," Annals of Neurology, vol. 53, no. 2, pp. 263-266, 2003.

[18] J. J. Eggermont, "Pathophysiology of tinnitus," Progress in Brain Research, vol. 166, pp. 19-543, 2007.

[19] D. De Ridder, A. B. Elgoyhen, R. Romo, and B. Langguth, "Phantom percepts: tinnitus and pain as persisting aversive memory networks," Proceedings of the National Academy of Sciences of the United States of America, vol. 108, no. 20, pp. 8075-8080, 2011.

[20] D. De Ridder, E. Verstraeten, K. Van Der Kelen et al., "Transcranial magnetic stimulation for tinnitus: influence of tinnitus duration on stimulation parameter choice and maximal tinnitus suppression," Otology and Neurotology, vol. 26, no. 4, pp. 616619, 2005.

[21] C. P. Lanting, E. De Kleine, H. Bartels, and P. Van Dijk, "Functional imaging of unilateral tinnitus using fMRI," Acta Oto-Laryngologica, vol. 128, no. 4, pp. 415-421, 2008.

[22] B. Langguth, "A review of tinnitus symptoms beyond 'ringing in the ears': a call to action," Current Medical Research and Opinion, vol. 27, no. 8, pp. 1635-1643, 2011.

[23] B. Langguth, T. Kleinjung, M. Landgrebe, D. de Ridder, and G. Hajak, "rTMS for the treatment of tinnitus: the role of neuronavigation for coil positioning," Neurophysiologie Clinique, vol. 40, no. 1, pp. 45-58, 2010.

[24] G. Frank, T. Kleinjung, M. Landgrebe et al., "Left temporal lowfrequency rTMS for the treatment of tinnitus: clinical predictors of treatment outcome- a retrospective study," European Journal of Neurology, vol. 17, no. 7, pp. 951-956, 2010.

[25] J. F. Piccirillo, D. Kallogjeri, J. Nicklaus et al., "Low-frequency repetitive transcranial magnetic stimulation to the temporoparietal junction for tinnitus: four-week stimulation trial," JAMA Otolaryngology-Head and Neck Surgery, vol. 139, no. 4, pp. 388-395, 2013.
[26] J. A. Henry, L. E. Roberts, D. M. Caspary, S. M. Theodoroff, and R. J. Salvi, "Underlying mechanisms of tinnitus: review and clinical implications," Journal of the American Academy of Audiology, vol. 25, no. 1, pp. 5-22, quiz 126, 2014.

[27] M. Lee, S. E. Kim, W. S. Kim et al., "Cortico-cortical modulation induced by $1-\mathrm{Hz}$ repetitive transcranial magnetic stimulation of the temporal cortex," Journal of Clinical Neurology, vol. 9, no. 2, pp. 75-82, 2013.

[28] U. Ziemann, F. Meintzschel, A. Korchounov, and T. V. Ilić, "Pharmacological modulation of plasticity in the human motor cortex," Neurorehabilitation and Neural Repair, vol. 20, no. 2, pp. 243-251, 2006.

[29] V. Di Lazzaro, F. Pilato, M. Dileone et al., "GABAA receptor subtype specific enhancement of inhibition in human motor cortex," Journal of Physiology, vol. 575, no. 3, pp. 721-726, 2006.

[30] P. Eichhammer, T. Kleinjung, M. Landgrebe, G. Hajak, and B. Langguth, "TMS for treatment of chronic tinnitusneurobiological effects," Progress in Brain Research, vol. 166, pp. 369-375, 2007.

[31] H. Burton, A. Wineland, M. Bhattacharya, J. Nicklaus, K. S. Garcia, and J. F. Piccirillo, "Altered networks in bothersome tinnitus: a functional connectivity study," BMC Neuroscience, vol. 13, no. 1, article 3, 2012.

[32] M. R. Laureano, E. T. Onishi, R. A. Bressan et al., "Memory networks in tinnitus: a functional brain image study," PLoS ONE, vol. 9, no. 2, article e87839, 2014.

[33] C. Plewnia, M. Reimold, A. Najib, G. Reischl, S. K. Plontke, and C. Gerloff, "Moderate therapeutic efficacy of positron emission tomography-navigated repetitive transcranial magnetic stimulation for chronic tinnitus: A Randomised, Controlled Pilot Study," Journal of Neurology, Neurosurgery and Psychiatry, vol. 78, no. 2, pp. 152-156, 2007. 

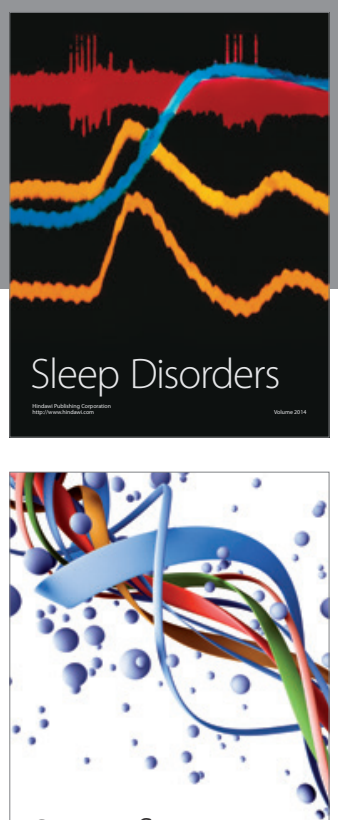

Scientifica
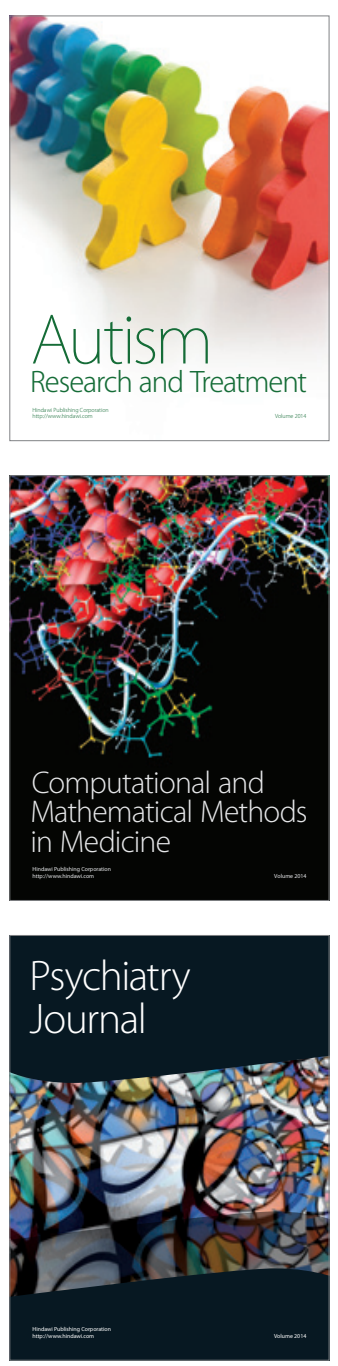
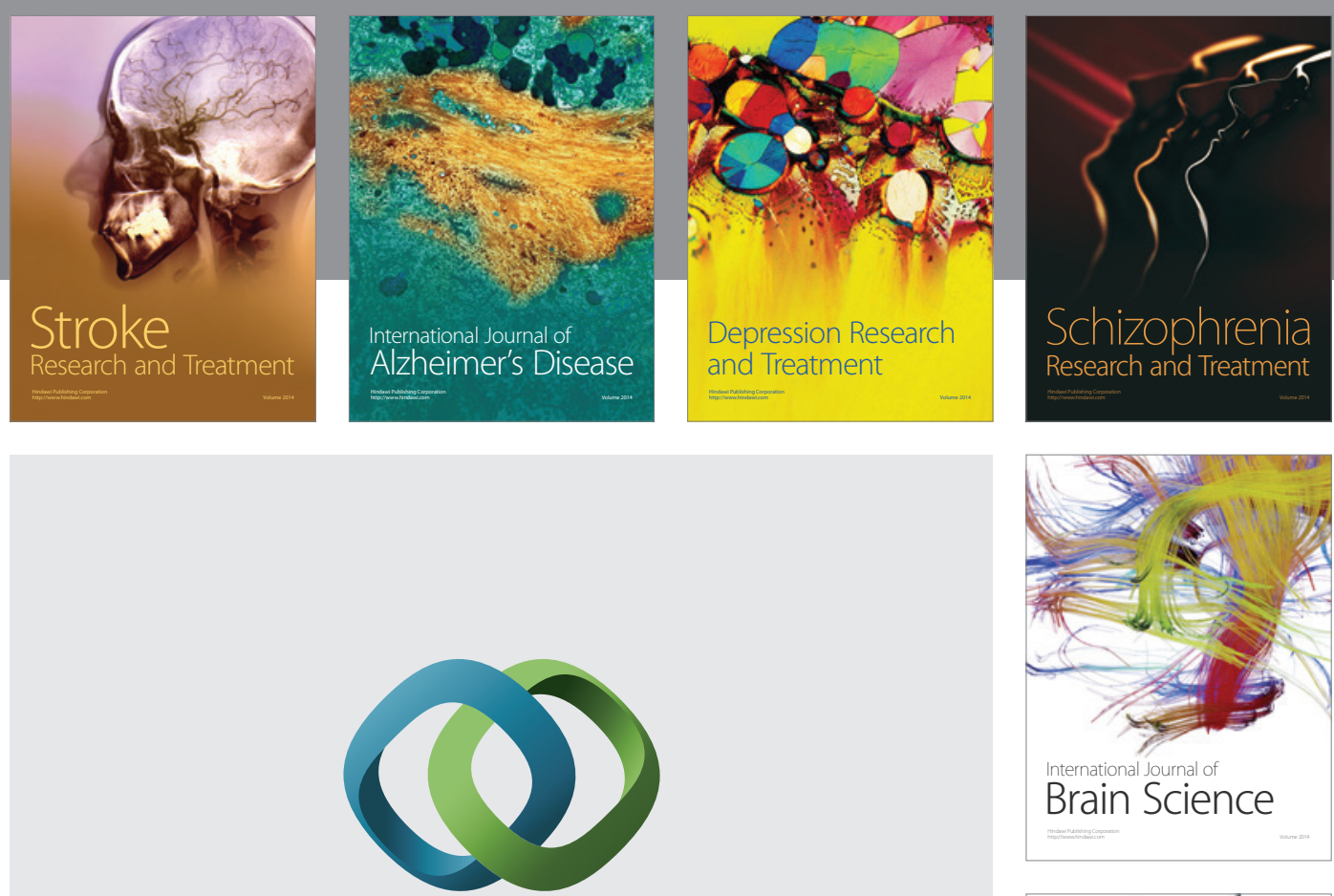

\section{Hindawi}

Submit your manuscripts at

http://www.hindawi.com
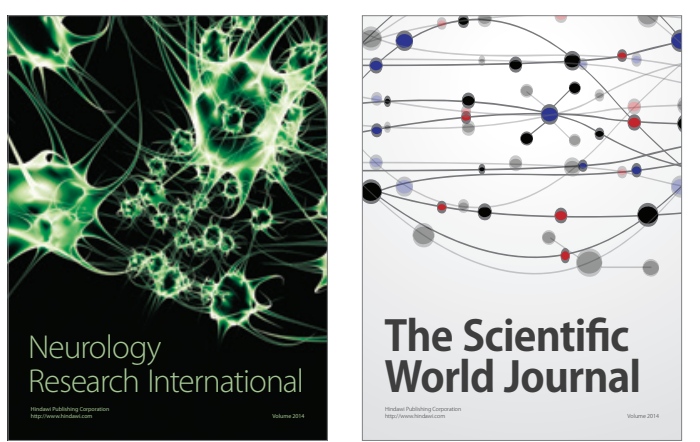

The Scientific World Journal

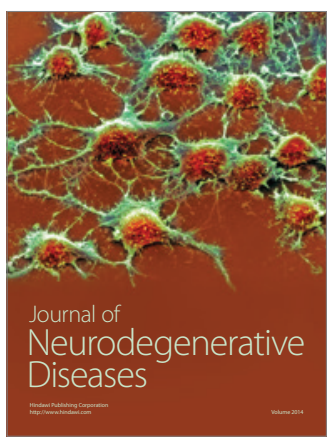

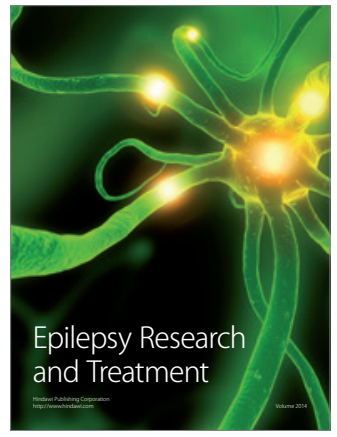

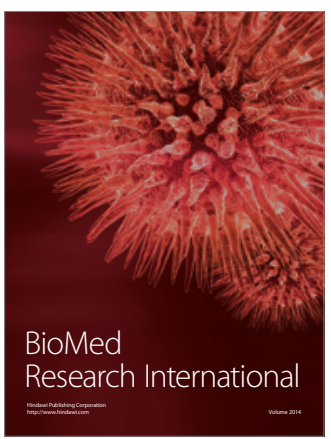

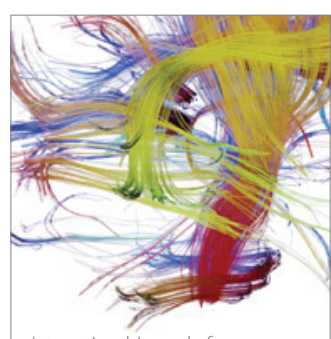

Brain Science

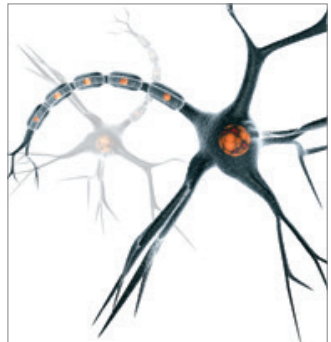

Neural Plasticity
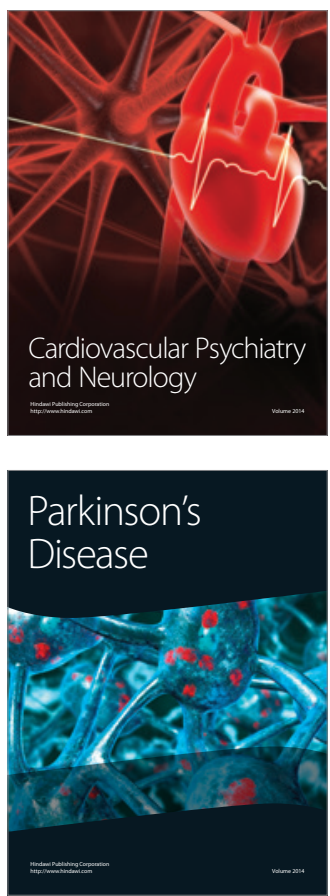